\title{
Observer agreement for diagnosis of colorectal lesions with analysis of the vascular pattern by image-enhanced endoscopy
}

Authors

Institutions
Carlos Eduardo Oliveira dos Santos ${ }^{1}$, Horácio Joaquin Perez² , Klaus Mönkemüller ${ }^{3}$, Daniele Malaman' ${ }^{1}$ César Vivian Lopes $^{4}$, Júlio Carlos Pereira-Lima ${ }^{4}$

Institutions are listed at the end of article. submitted

16. September 2014 accepted after revision 12. January 2015

\section{Bibliography}

DOI http://dx.doi.org/

10.1055/s-0034-1391667

Published online: 14.4.2015

Endosc Int Open 2015; 03:

E240-E245

(c) Georg Thieme Verlag KG

Stuttgart · New York

E-ISSN 2196-9736

\section{Corresponding author}

\section{César Vivian Lopes, MD PhD}

Rua Prof. Cristiano Fischer 668 / 1001

CEP 91410-000

Porto Alegre-RS

Brazil

Fax: +55-51-33388054

drcvlopes@gmail.com

\section{License terms}

Background/Aims: Image-enhanced endoscopy (IEE) can differentiate neoplastic from non-neoplastic colorectal lesions through indirect analysis of pit patterns and microvascular architecture. We evaluated the accuracy of Flexible Spectral Imaging Color Enhancement (FICE) in differentiating neoplastic from non-neoplastic lesions and observer agreement in the analysis of capillary pattern of colorectal lesions.

Methods: A prospective double-blind trial was conducted in two referral endoscopy centers. Vascular pattern was analyzed by IEE with magnification. Lesions were divided into two groups and examined separately by two experts. Examiners, blinded to each other's interpretations, switched groups and the lesions were reviewed. After 60 days, lesions were reevaluated.

\section{Introduction \\ $\nabla$}

Colonoscopy with resection of precursor lesions has led to a significant reduction in the incidence of colorectal cancer [1]. In addition to the adenoma-carcinoma sequence [2], it is important to recognize the pathway of de novo cancer (carcinoma without prior adenomatous tissue) [3-5], and the serrated pathway, where sessile serrated adenomas and traditional serrated adenomas are known to be precursors of cancer [4].

Colonoscopy is widely accepted as the gold standard for the diagnosis of colorectal lesions, and chromoendoscopy (CE) with indigo carmine or cresyl violet may help characterize the morphology of lesions, whose correct interpretation is very important in choosing the appropriate resection technique. CE is a powerful tool for the differentiation between neoplastic and non-neoplastic lesions, to predict the depth of neoplastic invasion, and in the diagnosis of residual tumors after endoscopic resection, which could improve the efficacy of the endoscopic procedure. This can be
Results: In total, 76 patients were referred to colonoscopy for colon cancer screening. Of 100 colorectal lesions, 88 were neoplastic ( 73 tubular adenomas, 10 tubulovillous adenomas, 1 villous adenoma, 2 serrated adenomas, 2 adenocarcinomas) and 12 were non-neoplastic (hyperplastic polyps). Mean diameter of the lesions was 6.7 $\mathrm{mm}$. Examiners 1 and 2 had 95\% accuracy. The interobserver kappa coefficient was 0.80 and the intraobserver kappa coefficient was 0.88 for examiner 1 and 0.73 for examiner 2 .

Conclusion: IEE with magnification is effective for real-time predictive histological diagnosis of colorectal lesions, with inter- and intraobserver agreement ranging from good to excellent.

achieved with CE using pit or capillary microvascular pattern analysis [6-10].

At the push of a button and with no need for dyes, image-enhanced endoscopy (IEE) has enabled us to obtain a real-time predictive histological diagnosis, both in the differential diagnosis between neoplastic and non-neoplastic lesions [11,12] and in the assessment of invasion depth of early cancer [13], through the analysis of surface (pitlike pattern) or capillary microvascular structures. Equipment-based IEE methods are represented by the Flexible Spectral Imaging Color Enhancement (FICE, Fujifilm), i-Scan (Pentax), and Narrow-Band Imaging (NBI, Olympus) systems. FICE and i-Scan systems are based on a computed spectral estimation technology that processes the reflected photons to reconstitute virtual images for a choice of different wavelengths of red, green, and blue signaling. The NBI system is based on modifying the bandwidth transmittance of spectral features using optical filters within the light source and a frame sequential lighting method. All technologies can enhance visualization of the mucosal surface structure, as well as the vascular 
meshwork, and help increase the visibility and characterization of neoplasms by improving contrast. This technology of advanced "dyeless" endoscopy has shown results similar to those of CE, especially when combined with magnification [14,15].

This study aimed to evaluate the accuracy and the inter- and intraobserver agreement of FICE with magnification in the differentiation of neoplastic from non-neoplastic colorectal lesions.

\section{Methods \\ $\nabla$}

Between September and November 2012, this prospective double-blind study analyzed 100 colorectal lesions in 76 consecutive patients (39 men and 37 women). The study was based on anonymous, blinded interpretation of electronically collected images in an ex vivo setting and would not result in any change of standard clinical care of the patients. The lesion was resected after being diagnosed and analyzed by FICE with magnification during the same procedure. The endoscopic images were eligible for inclusion in the study if the subjects were older than 40 years and their colonoscopy was being performed for colon cancer screening. Exclusion criteria were poor bowel preparation, incomplete colonoscopy, coagulopathy, presence of inflammatory bowel disease, polyposis syndrome, pregnancy, advanced cancer, failure to provide written informed consent, or patients with previous colonoscopy or surgical resection of the colon or rectum.

Initially, two groups of 50 consecutive lesions in 37 and 39 patients, respectively, were diagnosed and examined in real time separately by two experienced colonoscopists (CEOS and HJP) who have routinely used magnification and CE for over 10 years and performed more than 1500 colonoscopies with FICE. The two examiners then switched groups. The lesions were reviewed as high resolution images and FICE with magnification. The examiners were blinded to each other's interpretations. After 60 days, all lesions were blindly reevaluated by each of the two endoscopists to check for inter- and intraobserver agreement. Histological analysis served as the gold standard.

Bowel preparation consisted of a 1-day clear liquid diet, with intake of $1 \mathrm{~L}$ of $10 \%$ mannitol solution on the morning of the examination. Procedures were performed with the patient under conscious sedation (intravenous midazolam and meperidine or fentanyl).

The Paris classification was used as a reference to determine the morphology of lesions [16]. Lesion size was estimated at real time colonoscopy using an open biopsy forceps. The location was estimated from anatomic landmarks.

All lesions were diagnosed under white light, cleaned with tap water when necessary, and subsequently analyzed with a high resolution, magnification $(\times 60-100)$ colonoscope (Fujinon 590ZW5, Fujifilm Corp., Saitama, Japan), equipped with the EPX 4400 processor and FICE system, which enabled examination of wavelengths from 400 to $700 \mathrm{~nm}$ at 5-nm intervals. No chromoscopy with indigo carmine was performed before FICE. In this study, the following wavelengths were standardized: R550(2), $\mathrm{G} 500$ (3) and B470(2), and used for real-time evaluation of the capillary pattern. The analysis was based on the Teixeira classification [17], which has five subtypes: type I is the normal pattern, with thin capillary vessels with a linear shape; type IIa presents hypovascularity or some capillaries of a thicker diameter, curved or straight but uniform, without dilatations ( $\bullet$ Fig. 1); type III exhibits numerous capillaries of thinner diameter, irregular and tortuous, with frequent point dilatations, and tapering like a spir-

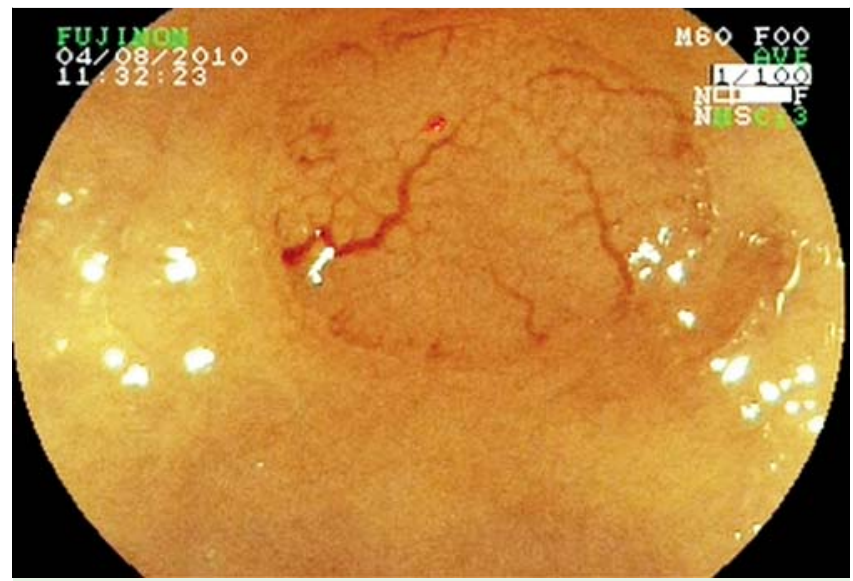

Fig. 1 Type II (Teixeira classification): Lesion with capillaries of a thicker diameter, curved, without dilatations. Hyperplastic polyp.

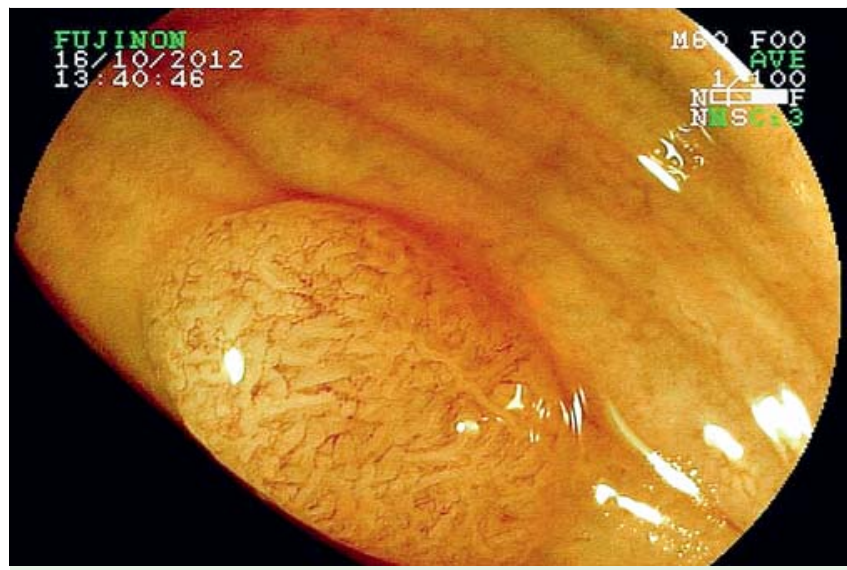

Fig. 2 Type III (Teixeira classification): Lesion showing several fine and irregularly distributed tortuous capillaries, with some points of dilatation, and tapering like a spiral shape. Tubular adenoma.

al shape, showing a remarkable periglandular arrangement ( $\bullet$ Fig. 2); type IVa presents numerous long, spiral, or straight vessels with a thicker diameter, and sparse dilatations, surrounding the villous glands ( Fig.3); type V shows pleomorphism of capillaries and abnormal distribution and arrangement ( $\bullet$ Fig.4). All lesions were removed endoscopically with biopsy forceps, by snare polypectomy, or by endoscopic mucosal resection. Specimens were fixed in $10 \%$ formalin and subsequently examined using hematoxylin and eosin staining by two pathologists, who were blinded to the endoscopic results and who followed the guidelines of the World Health Organization classification of colorectal tumors [18]. In the case of multiple lesions in the same patient, each lesion was identified individually and placed in different flasks.

The study was approved by the institutional review board of each center and conducted in accordance with the principles of the Declaration of Helsinki, and written informed consent was obtained from all patients before endoscopy.

\section{Statistical analysis}

Data were analyzed using Stata 11.1 Statistical Package (StatCorp LP, College Station, TX, United States). Numerical variables were expressed as mean and standard deviation, and categorical vari- 


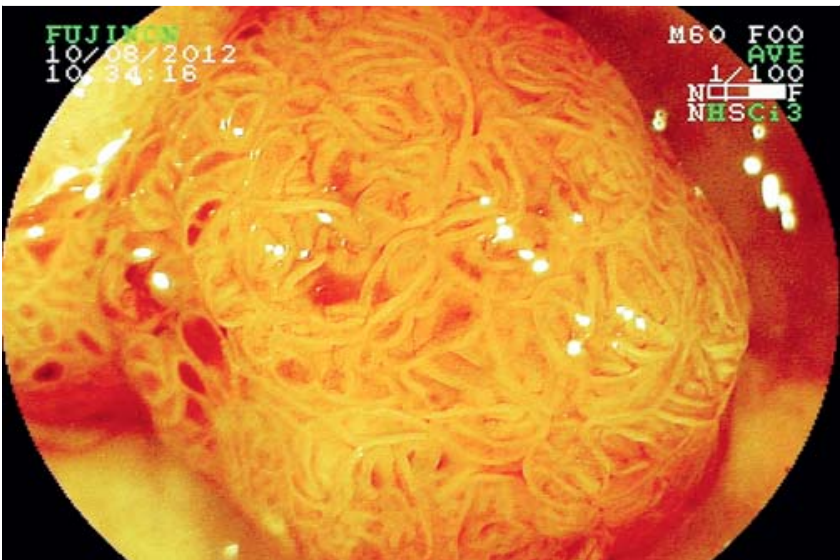

Fig. 3 Type IV (Teixeira classification): Lesion showing numerous, elongated, and spiral-shaped capillaries around villous glands. Tubulovillous adenoma.

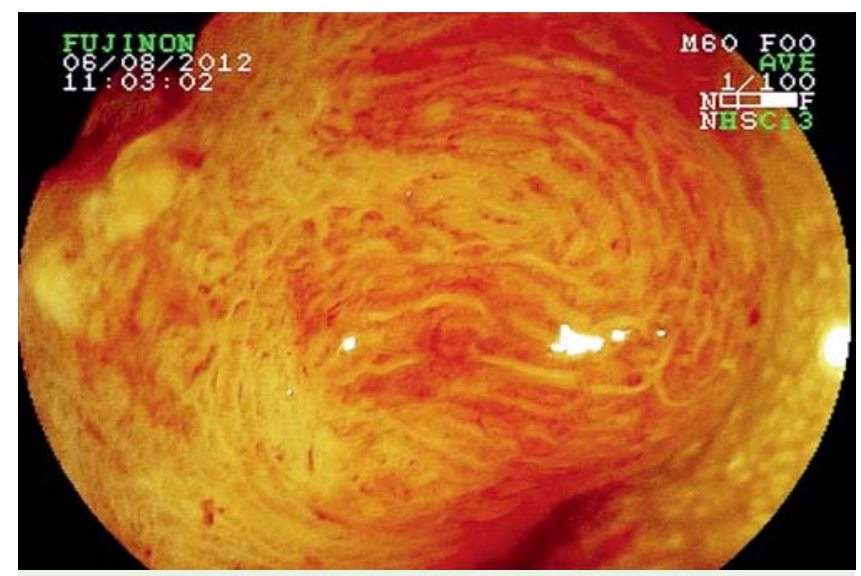

Table 1 Characteristics of patients and colorectal lesions.

\begin{tabular}{|l|l|}
\hline Variable & $\mathbf{n}$ \\
\hline Sex & $37(48.7 \%)$ \\
\hline Female & $39(51.3 \%)$ \\
\hline Male & $58( \pm 12.6)$ \\
\hline Mean patient age (years) & $6.7($ range $2-30)$ \\
\hline Mean lesion size $(\mathrm{mm})$ & \\
\hline Histopathology & 88 \\
\hline Neoplastic & 73 \\
\hline Tubular adenoma & 10 \\
\hline Tubulovillous adenoma & 1 \\
\hline Villous adenoma & 2 \\
\hline Serrated adenoma & 2 \\
\hline Adenocarcinoma & 12 \\
\hline Non-neoplastic & 12 \\
\hline Hyperplastic polyp & 36 \\
\hline Macroscopic type & 64 \\
\hline Flat & 36 \\
\hline Protruding & 31 \\
\hline Macroscopic classification & 3 \\
\hline Nonpolypoid & 2 \\
\hline $0-$ Ila & 64 \\
\hline $0-$ Ila +llc & 49 \\
\hline $0-$ Ilc +lla & 12 \\
\hline Polypoid & 3 \\
\hline $0-$ Is & 50 \\
\hline $0-$ Isp & \\
\hline $0-$ Ip & \\
\hline Location & Right side of the colon \\
\hline Left side of the colon & \\
\hline
\end{tabular}

Fig. 4 Type V (Teixeira classification): Lesion showing numerous, thick, heterogeneous vessels with disorganized microvascular architecture.

ables as percentage. The Student's $t$-test was used for the bivariate analysis of mean age and the nonparametric Mann - Whitney test was used for the bivariate analysis of mean lesion size owing to the asymmetric distribution of this variable. Fisher's exact test was used for the bivariate analysis of histopathology (neoplastic or non-neoplastic lesions). A $P$-value of less than 0.05 was considered to be statistically significant. Based on preliminary experience, supposing an accuracy of $90 \%$ for the digital chromoendoscopy in differentiating neoplastic from non-neoplastic colorectal lesions, with a $P$-value of 0.05 , a power of 0.80 , and a margin of error of $6 \%$, a total of 96 patients should be enrolled in the study. The reproducibility coefficients were analyzed by the kappa agreement coefficient. Inter- and intraobserver agreement values were classified as follows: poor $(\leq 0.20)$; slight $(0.21-$ 0.40 ); moderate (0.41 - 0.60); substantial (0.61 - 0.80); and excellent $(0.81-1.00)$. Inter- and intraobserver diagnostic values vs. histopathological analysis were compared for sensitivity, specificity, positive predictive value, and negative predictive value.

\section{Results}

$\nabla$

The characteristics of patients and lesions are shown in $\bullet$ Table 1. There was no statistically significant difference in the prevalence of neoplastic versus non-neoplastic lesions with regard to mac-

roscopic analysis, location, or size. Ten lesions were adenomas with high grade dysplasia or adenocarcinomas; of these, seven were polypoid lesions and three were nonpolypoid depressed lesions, the latter showing a significantly smaller mean size (5.0 vs. $17.0 \mathrm{~mm} ; P=0.04)$, accounting for two adenomas and one adenocarcinoma.

Of 100 colorectal lesions, 88 were neoplastic; of these, 73 were tubular adenomas, 10 were tubulovillous adenomas, 1 was a villous adenoma, 2 were traditional serrated adenomas (type 0 - Is with $7 \mathrm{~mm}$ in the descending colon, and type 0 - Isp with $10 \mathrm{~mm}$ in the rectum, both capillary pattern type IV), and 2 were adenocarcinomas. All 12 non-neoplastic lesions were hyperplastic polyps. The mean number of lesions per patient was 1.3 , and of neoplasms per patient was 1.2. Mean lesion size was $6.7 \mathrm{~mm}$, ranging from 2 to $30 \mathrm{~mm}$ in diameter, with no statistically significant difference between neoplastic and non-neoplastic lesions ( 4.3 vs. $7.1 \mathrm{~mm} ; P=0.07$ ). Nonpolypoid lesions were significantly smaller than polypoid lesions ( 4.0 vs. $8.3 \mathrm{~mm}$; $P<0.001$ ). Eightyeight lesions were $\leq 10 \mathrm{~mm}$ in diameter. Patients with neoplasms were older than patients with non-neoplastic lesions (59.2 vs. 49.9 years; $P=0.03$ ).

Regarding morphology, 36 lesions were classified as nonpolypoid lesions (31, type 0 - IIa; 3, type 0 - Ila + IIc; and 2, type 0 - IIc + IIa) and 64 as polypoid lesions (49, type 0 -Is; 12 , type 0 - Isp; and 3, type 0 - Ip).

In the analysis of all 100 lesions, examiners 1 and 2 had an accuracy of $95 \%$ ( $95 \% \mathrm{CI} 90.6-99.4$ ) and for the other diagnostic criteria, values were: sensitivity of $97.7 \%$ and $98.9 \%$, specificity of $75 \%$ 
Table2 Diagnostic criteria for the two observers in histological prediction.

\begin{tabular}{|lll|}
\hline & Observer 1 & Observer 2 \\
\hline Accuracy, \% & 95 & 95 \\
\hline Sensitivity, \% $(95 \% \mathrm{Cl})$ & $97.7(92.0-99.7)$ & $98.9(93.8-100)$ \\
\hline Specificity, \% $(95 \% \mathrm{Cl})$ & $75.0(42.8-94.5)$ & $66.7(34.9-90.0)$ \\
\hline PPV, \% $(95 \% \mathrm{Cl})$ & $96.6(90.5-99.3)$ & $95.6(89.1-98.8)$ \\
\hline NPV, \% $(95 \% \mathrm{Cl})$ & $81.8(48.2-97.7)$ & $88.9(51.8-99.7)$ \\
\hline
\end{tabular}

$95 \% \mathrm{Cl}, 95 \%$ confidence interval; PPV, positive predictive value; NPV, negative predictive value.

and $66.7 \%$, positive predictive value (PPV) of $96.6 \%$ and $95.6 \%$, and negative predictive value (NPV) of $81.8 \%$ and 88.9 , respectively ( Table 2). For nonpolypoid and polypoid lesions, the accuracies were $100 \%$ vs. $92.2 \%$ and $97.7 \%$ vs. $93.8 \%$, respectively. Examiners 1 and 2 showed an accuracy of $94.3 \%$ (95\%CI 89.499.2) when considering only the 88 lesions $\leq 10 \mathrm{~mm}$ in diameter, sensitivity of $97.4 \%$ and $98.7 \%$, specificity of $75.0 \%$ and $66.7 \%$, PPV of $96.6 \%$ and $94.9 \%$, and NPV of $81.8 \%$ and $88.9 \%$, respectively. In the evaluation of lesions $\leq 5 \mathrm{~mm}$ in size, both examiners had an accuracy of $92.7 \%$ (95\%CI $85.7-99.7)$.

\section{Interobserver agreement}

In the analysis of the five capillary pattern types in all 100 lesions, the interobserver kappa coefficient was 0.80 (95\%CI 0.75-0.85). For the 88 small lesions ( $\leq 10 \mathrm{~mm}$ ), the kappa coefficient was 0.88 (95\%CI $0.73-1.00)$.

When capillary pattern types I and II (non-neoplastic lesions) and types III-V (neoplasms) were compared, the kappa coefficient was 0.89 (95\%CI $0.74-1.00)$. When analyzing only lesions $\leq 10 \mathrm{~mm}$ in diameter for predictive histological diagnosis (neoplasm vs. hyperplastic polyp), the kappa coefficient was also 0.89 (95\%CI $0.73-1.00)$. For the 55 lesions $\leq 5 \mathrm{~mm}$, the kappa coefficient was 0.84 (95\%CI $0.62-1.00)$, and for the 45 lesions $>5 \mathrm{~mm}$, the kappa coefficient was 1.00 .

The kappa coefficient was 0.91 (95\%CI $0.73-1.00$ ) for nonpolypoid lesions and 0.85 (95\%CI $0.56-1.00)$ for polypoid lesions. The kappa coefficient for examiners 1 and 2 for prediction of the histological type was 0.75 vs. 0.74 , with similar values for small lesions ( $\leq 10 \mathrm{~mm}$ ), $k=0.75$ vs. 0.73 . For lesions $\leq 5 \mathrm{~mm}$, the kappa coefficient was 0.71 and 0.67 , respectively, and for lesions $>5$ $\mathrm{mm}$, kappa was 0.85 for both examiners.

\section{Intraobserver agreement}

In the analysis of the five capillary pattern types in all 100 lesions, the intraobserver kappa coefficient was 0.88 (95\%CI $0.83-0.94$ ) for examiner 1 and 0.73 (95\%CI $0.65-0.81$ ) for examiner 2. For small lesions $(\leq 10 \mathrm{~mm})$, the kappa coefficient was $0.82(95 \% \mathrm{CI}$ $0.76-0.90)$ and $0.67(95 \% \mathrm{Cl} 0.57-0.74)$, respectively.

In the predictive histological diagnosis between neoplasm and hyperplastic polyp, the intraobserver kappa coefficient was 0.95 (95\%CI $0.83-0.94)$ and 0.70 (95\%CI $0.65-0.81)$, with similar results for small lesions ( $\leq 10 \mathrm{~mm}): k=0.95$ (95\%CI $0.84-1.00)$ and $\kappa=0.69$ (95\%CI $0.46-0.92$ ). For lesions $\leq 5 \mathrm{~mm}$, the kappa coefficient was $0.92(0.77-1.00)$ for examiner 1 and $0.71(0.45-0.97)$ for examiner 2, while for lesions $>5 \mathrm{~mm}$ : $k=1.00$ and $0.64(0.18-$ $1.00)$, respectively.

For examiner 1 and 2, the kappa coefficient was 0.85 (95\%Cl $0.67-1.00)$ and 0.78 (95\%CI 0.65-0.94), respectively, for nonpolypoid lesions, and $0.90(95 \% \mathrm{CI} 0.82-0.97)$ and $0.70(95 \% \mathrm{CI}$ $0.58-0.81$ ) for polypoid lesions.

\section{Discussion}

7

Image-enhanced endoscopy (IEE) with high magnification is an effective technology for real-time predictive histological diagnosis of colorectal lesions, with inter- and intraobserver agreements ranging from good to excellent. Previous studies using CE techniques such as NBI and i-Scan have demonstrated that these methods are useful to characterize colorectal lesions. However, there is still a lack of studies using FICE. In addition, most previous studies have focused on analyzing the mucosal pit pattern and few have focused on the capillary pattern. Whereas the pit pattern represents changes on the mucosal surface, the capillary pattern reflects changes in the number, morphology, and distribution of the fine blood vessels.

Rastogi et al. [19] analyzed 65 polyps (38 adenomas and 27 hyperplastic polyps) and reported a kappa value for interobserver agreement of 0.57 for polyp surface pattern and of 0.63 for prediction of histological type. The kappa value for intraobserver agreement ranged from 0.60 to 0.79 for polyp surface pattern and from 0.61 to 0.87 for predictive diagnosis. The accuracy ranged from $80 \%$ to $86 \%$ in the first reading and from $85 \%$ to 91 $\%$ in the second reading of images. In the series by Huang et al. [20], six experienced endoscopists used the Kudo classification $[21,22]$ and CE for pit pattern analysis, with inter- and intraobserver kappa values of 0.72 and 0.81 , respectively; for predictive histological diagnosis, kappa values were 0.77 and 0.86 , respectively.

East et al. [23] compared the use of CE and IEE (NBI) with magnification in pit pattern analysis of 33 polyps, according to the Kudo classification. The results were different for 12 of 33 polyps for examiner 1 and 20 of 33 polyps for examiner 2, with a combined kappa of 0.23 . The authors concluded that the Kudo classification should be modified and revalidated for use with IEE. However, in our previous study of 111 small lesions ( $\leq 10 \mathrm{~mm}$ ), we obtained $90.1 \%$ accuracy using the Kudo classification for pit pattern analysis with IEE [24]. Although Kudo's classification was not designed to be used with IEE, other series have shown similar results $[11,12,25]$. Nevertheless, this contradictory result indicates that the pit pattern classification may need further refinement and more studies focused on capillary analysis are warranted.

A previous study examining the capillary pattern of colorectal lesions yielded a diagnostic accuracy of $98.3 \%$ in differentiating neoplastic from non-neoplastic lesions, and the interobserver kappa coefficient was 0.85 [17]. However, a multicenter study involving nine experts from three different countries (Brazil, Japan, and Venezuela) to evaluate 45 lesions reported an accuracy of $82.2 \%$ to $100 \%$ with kappa values ranging from 0.41 to 0.84 for the prediction of histological diagnosis, and an interobserver kappa coefficient of 0.64 [26]. These data emphasize the need for additional studies such as this one. In our previous studies comparing standard CE with IEE, the accuracy to predict histology using IEE ranged from $92.8 \%$ to $93.6 \%$ [24,27]. Similar to the Kudo classification, we also believe that the capillary meshwork classification should be simplified in order to become more userfriendly.

In our study, we used the currently accepted Teixeira classification for capillary pattern analysis, differentiating the pattern for non-neoplastic lesions (type I and II) from the pattern for neoplastic lesions (type III-V), with type $\mathrm{V}$ being highly suggestive of adenocarcinoma. We obtained an accuracy of $95 \%$ for both examiners, and the kappa value for interobserver agreement was 0.80 for capillary pattern analysis and 0.89 for prediction of his- 
topathology (neoplasm vs. hyperplastic polyp), including small lesions. The kappa value for intraobserver agreement was 0.88 and 0.73 for capillary pattern analysis, and 0.95 and 0.70 for predictive histological analysis (neoplasm vs. hyperplastic polyp) for the two examiners. In the study of lesions $\leq 5 \mathrm{~mm}$ in size, both examiners had an accuracy of $92.7 \%$. In the predictive histological diagnosis, the interobserver kappa coefficient was 0.84 for lesions $\leq 5 \mathrm{~mm}$, and 1.00 for lesions $>5 \mathrm{~mm}$. The intraobserver kappa coefficient for lesions $\leq 5 \mathrm{~mm}$ was 0.92 for examiner 1 and 0.71 for examiner 2 , while for lesions $>5 \mathrm{~mm}$, the kappa values were 1.00 and 0.64 , respectively.

Although some experts may argue that magnification is not widely available, we believe that a high diagnostic yield and careful endoscopic characterization are essential when dealing with potentially malignant lesions. Indeed, if a method is used to decide whether to leave a lesion after performing a biopsy or to resect it, a high diagnostic accuracy is needed.

We want to emphasize that a large number of small lesions are hyperplastic polyps, which, because they have no malignant potential, need not be removed. Endoscopic resection should be reserved only for neoplastic lesions. Nevertheless, studies have shown that about $30 \%$ of colorectal carcinomas develop through the serrated neoplasia pathway. Several lesions previously classified as hyperplastic polyps, and therefore thought to be harmless, have been reclassified as sessile serrated adenomas, establishing their place in the serrated pathway and underscoring their malignant potential. Sessile serrated adenomas are located preferentially in the right side of the colon, with a flat morphology, and with a type II-O pit pattern (Kimura classification) [28]. Traditional serrated adenomas, however, are often located in the left side of the colon, are polypoid lesions, and have a villous architecture and a type IV pit pattern [29]. The mean size of sessile serrated adenomas is greater than that of traditional serrated adenomas. It is recommended that all serrated lesions proximal to the sigmoid colon and all serrated lesions in the rectosigmoid colon greater than $5 \mathrm{~mm}$ in diameter should be completely removed [4].

In this study, we had two small traditional serrated adenomas, polypoid lesions located in the left side of the colon, which showed a type IV pit pattern. The fact that the histological diagnostic criteria for these lesions are not widely known among Brazilian pathologists may have influenced these results. Also important are depressed lesions, which have a more aggressive progression than polypoid lesions and may invade the submucosa early, even when they are small or diminutive lesions. In this study, of 100 lesions analyzed, we found five depressed lesions, and of 10 lesions histologically classified as adenomas with high grade dysplasia or adenocarcinomas, $30 \%$ were depressed lesions, of which one was an intramucosal adenocarcinoma 10 $\mathrm{mm}$ in size. Depressed lesions had a significantly smaller mean size than polypoid lesions $(P=0.04)$.

Accuracy results have been shown to have a direct relationship with lesion size, i.e. the smaller the lesion, the worse the accuracy values [25]. The vessels of the microvascular structure of the normal colorectal epithelium are 5 to $10 \mu \mathrm{m}$ in diameter. Visualization of the capillary pattern of lesions smaller than $5 \mathrm{~mm}$ in diameter is not easy, which may explain these poor results. However, in our previous study of diminutive colorectal lesions, we had $92.6 \%$ accuracy, with a kappa value of 0.81 , suggesting that experience using the method may be important [30].

We would like to acknowledge the potential limitations of our study. First, the endoscopists performing the procedures were well versed in the IEE technique. Nonetheless, advanced imaging is becoming the standard of care and all endoscopists participating in colorectal cancer screening should be well trained in polyp recognition and characterization methods. Second, we had only 100 lesions and two endoscopists involved in the study. However, our study included more lesions than previous studies. In addition, we focused on performing the intraobserver agreement 60 days after the initial reading, in an attempt to limit potential bias in the readings. Finally, this and other current endoscopic techniques cannot distinguish between hyperplastic and serrated lesions. However, our study was designed to analyze the accuracy to interpret common lesions using this technology. Thus, we strongly believe we reached our goal of objectively testing a relatively new endoscopic imaging technology.

In conclusion, IEE (FICE) combined with high magnification for capillary pattern analysis is an effective technology for real-time predictive histological diagnosis of colorectal lesions. We achieved substantial interobserver agreement for the analysis of microvascular structure and excellent agreement for predictive histological diagnosis, while intraobserver agreement ranged from good to excellent both for capillary pattern analysis and for differential diagnosis between neoplastic and non-neoplastic lesions.

\section{Competing interests: None}

\section{Institutions}

1 Department of Digestive Endoscopy and Gastroenterology, Santa Casa Hospital, Bagé, Brazil

2 Department of Digestive Endoscopy, Universidade Federal de Santa Catarina, Florianópolis, Brazil

${ }^{3}$ Basil Hirschowitz Endoscopic Center of Excellence, University of Alabama, Birmingham, USA

${ }^{4}$ Department of Gastroenterology, Santa Casa Hospital, Porto Alegre, Brazil

\section{References}

1 Zauber AG, Winawer SJ, O'Brien MJ et al. Colonoscopic polypectomy and long-term prevention of colorectal-cancer deaths. NEJM 2012; 366: 687-696

2 Morson BC. The polyp-cancer sequence in the large bowel. Proc R Soc Med 1974; 67: 451 - 457

3 Matsuda T, Saito Y, Hotta $K$ et al. Prevalence and clinicopathological features of nonpolypoid colorectal neoplasms: should we pay more attention to identifying flat and depressed lesions? Dig Endosc 2010; 22: 57-S62

4 Rex DK, Ahnen DJ, Baron JA et al. Serrated lesions of the colorectum: review and recommendations from an expert panel. Am J Gastroenterol 2012; 107: 1315-1329

5 Sano $Y$, Tanaka S, Teixeira $C R$ et al. Endoscopic detection and diagnosis of 0-IIc neoplastic colorectal lesions. Endoscopy 2005; 37: 261-267

$6 \mathrm{Su} M Y, H o$ YP, Chen PC et al. Magnifying endoscopy with indigo carmine contrast for differential diagnosis of neoplastic and nonneoplastic colonic polyps. Dig Dis Sci 2004; 49: 1123-1127

7 Zanoni EC, Cutait $R$, Averbach $M$ et al. Magnifying colonoscopy: interobserver agreement in the assessment of colonic pit patterns and its correlation with histopathological findings. Int J Colorectal Dis 2007; 22: $1383-1388$

8 Matsuda T, Fujii T, Saito $Y$ et al. Efficacy of the invasive/non-invasive pattern by magnifying chromoendoscopy to estimate the depth of invasion of early colorectal neoplasms. Am J Gastroenterol 2008; 103: $2700-2706$

9 Hurlstone DP, Cross SS, Brown S et al. A prospective evaluation of highmagnification chromoscopic colonoscopy in predicting completeness of EMR. Gastrointest Endosc 2004; 59: $642-650$

10 Santos CE, Malaman D, Pereira-Lima JC. Endoscopic mucosal resection in colorectal lesion: a safe and effective procedure even in lesions larger than $2 \mathrm{~cm}$ and in carcinomas. Arq Gastroenterol 2011; 48: 242 247 
11 Hirata M, Tanaka S, Oka $S$ et al. Magnifying endoscopy with narrow band imaging for diagnosis of colorectal tumors. Gastrointest Endosc 2007; 65: 988 - 995

12 Tischendorf JJ, Wasmuth HE, Koch A et al. Value of magnifying chromoendoscopy and narrow band imaging (NBI) in classifying colorectal polyps: a prospective controlled study. Endoscopy 2007; 39: 10921096

13 Kanao H, Tanaka S, Oka S et al. Narrow-band imaging magnification predicts the histology and invasion depth of colorectal tumors. Gastrointest Endosc 2009; 69: 631 -639

$14 \mathrm{Su}$ MY, Hsu CM, Ho YP et al. Comparative study of conventional colonoscopy, chromoendoscopy, and narrow band imaging systems in differential diagnosis of neoplastic and non-neoplastic colonic polyps. Am J Gastroenterol 2006; 101: 2711-2716

15 Hoffman A, Kagel C, Goetz M et al. Recognition and characterization of small colonic neoplasia with high-definition colonoscopy using i-Scan is as precise as chromoendoscopy. Dig Liver Dis 2010; 42: 45-50

16 The Paris endoscopic classification of superficial neoplastic lesions. Gastrointest Endosc 2003; 58: 3-S43

17 Teixeira CR, Torresini RS, Canali C et al. Endoscopic classification of the capillary-vessel pattern of colorectal lesions by spectral estimation technology and magnifying imaging. Gastrointest Endosc 2009; 69: $750-756$

18 Hamilton SR, Aaltonen LA editors World Health Organization classification of tumours: Pathology and genetics of tumours of the digestive system. Lyon: IARC Press 2000: 104-119

19 Rastogi A, Pondugula K, Bansal A et al. Recognition of surface mucosal and vascular patterns of colon polyps by using narrow-band imaging: interobserver and intraobserver agreement and prediction of polyp histology. Gastrointest Endosc 2009; 69: 716-722

20 Huang $Q$, Fukami $N$, Kashida $\mathrm{H}$ et al. Interobserver and intra-observer consistency in the endoscopic assessment of colonic pit patterns. Gastrointest Endosc 2004; 60: 520-526
21 Kudo S, Hirota S, Nakajima T et al. Colorectal tumours and pit pattern. J Clin Pathol 1994; 47: 880-885

22 Kudo S, Tamura S, Nakajima T et al. Diagnosis of colorectal tumours lesions by magnifying endoscopy. Gastrointest Endosc 1996; 44: 8-14

23 East JE, Suzuki N, Saunders BP. Comparison of magnified pit pattern interpretation with narrow band imaging versus chromoendoscopy for diminutive colonic polyps: a pilot study. Gastrointest Endosc 2007; 66: $310-316$

24 dos Santos CE, Lima JC, Lopes CV et al. Computerized virtual chromoendoscopy versus indigo carmine chromoendoscopy combined with magnification for diagnosis of small colorectal lesions: a randomized and prospective study. Eur J Gastroenterol Hepatol 2010; 22: 1364 1371

25 Yoshida $N$, Naito $Y$, Inada $Y$ et al. The detection of surface patterns by flexible spectral imaging color enhancement without magnification for diagnosis of colorectal polyps. Int J Colorectal Dis 2012; 27: 605 611

26 Teixeira $C R$, Oliveira $L A$, Saito $Y$ et al. Worldwide multicenter interobserver validation of a novel endoscopic classification of the capillary vessel pattern of colorectal lesions. Gut 2010; 59: A72

27 Santos CE, Pereira-Lima JC, Lopes CV et al. Comparative study between $\mathrm{MBI}\left(\mathrm{FICE}^{\circledR}\right)$ and magnification chromoendoscopy with indigo carmine in the differential diagnosis of neoplastic and non-neoplastic colorectal lesions. Arq Gastroenterol 2009; 46: 111 - 115

28 Kimura T, Yamamoto E, Yamano HO et al. A novel pit pattern identifies the precursor of colorectal cancer derived from sessile serrated adenoma. Am J Gastroenterol 2012; 107: 460 - 469

29 Ishigooka S, Nomoto M, Obinata $N$ et al. Evaluation of magnifying colonoscopy in the diagnosis of serrated polyps. World J Gastroenterol 2012; 18: $4308-4316$

30 dos Santos CE, Malaman D, Lopes CV et al. Digital chromoendoscopy for diagnosis of diminutive colorectal lesions. Diagn Ther Endosc 2012; 2012: 279521 